\title{
Pratiques
}

Linguistique, littérature, didactique

187-188 | 2020

Enseignement du texte littéraire dans l'espace

francophone: pratiques, formation, recherche

\section{La séance de lecture de l'image dans la séquence des professeurs stagiaires de français : représentations et choix méthodologiques.}

The image reading session in the sequence of trainee French teachers:

representations and methodological choices.

Bénédicte Duvin-Parmentier

\section{(2) OpenEdition}

Journals

Édition électronique

URL : https://journals.openedition.org/pratiques/9337

DOI : $10.4000 /$ pratiques.9337

ISSN : 2425-2042

Éditeur

Centre de recherche sur les médiations (CREM)

Référence électronique

Bénédicte Duvin-Parmentier, «La séance de lecture de l'image dans la séquence des professeurs stagiaires de français : représentations et choix méthodologiques. », Pratiques [En ligne], 187-188| 2020, mis en ligne le 12 décembre 2020, consulté le 21 juillet 2021. URL : http://

journals.openedition.org/pratiques/9337 ; DOI : https://doi.org/10.4000/pratiques.9337

Ce document a été généré automatiquement le 21 juillet 2021.

(c) Tous droits réservés 


\section{La séance de lecture de l'image dans la séquence des professeurs stagiaires de français : représentations et choix méthodologiques.}

The image reading session in the sequence of trainee French teachers:

representations and methodological choices.

Bénédicte Duvin-Parmentier

\section{Introduction}

1 Les programmes de français depuis 1985 accordent une place croissante aux arts visuels et en particulier à l'image, au cinéma ou encore à la bande dessinée. Cependant, peu de recherches analysent le statut et la fonction de l'image dans la discipline. De nombreuses questions demeurent : l'image est-elle présente en français « pour venir au secours de la complexité de la littérature » (Gabathuler, 2013 ; 2016) ? Quels outils sont utilisés par les enseignants ? Et que signifie «lire» une image quand les outils préconisés relèvent généralement des pratiques littéraires (Massol, Duvin-Parmentier \& Claude, 2020)?

2 La «lecture» de l'image telle qu'elle est recommandée dans les programmes de français pour le collège et le lycée est essentiellement appréhendée sous l'angle sémiologique, dans une perspective structurale. Lorsqu'il est question de développer des compétences pour la décoder, comment prend-on alors en compte l'expression de la sensibilité du spectateur? Quelle part le langage occupe-t-il au moment de la réception de l'œuvre d'art? J.-C. Chabanne $(2013 ; 2016$; 2017) montre que l'image existe à travers la multiplicité de discours variés qui la traversent et que la lire, c'est en 
rendre compte par la parole. Ainsi souligne-t-il l'importance de repenser le dialogue fécond entre didactique du français et didactique des arts.

3 Toutes ces interrogations trouvent leurs origines devant le constat, d'une part, de la difficulté exprimée par les professeurs stagiaires de lettres quant à leurs représentations de la «lecture " de l'image dans l'organisation de la séquence de lettres, d'autre part, de l'existence d'un point aveugle en didactique concernant la représentation que les enseignants ont de la lecture de l'image en lien avec l'analyse du texte littéraire.

Cette étude cherche à documenter les représentations des professeurs stagiaires de lettres à travers leurs choix méthodologiques afin de mettre en œuvre un cahier des charges sur ce que pourrait être l'analyse de l'image dans la séquence de français en formation initiale et d'en mesurer les effets sur les pratiques des professeurs débutants. Notons que ces derniers semblent parfois, si l'on en croit leurs déclarations, en butte à certaines difficultés récurrentes et s'interrogent notamment sur le brouillage des frontières disciplinaires et la pertinence de l'exploitation de l'image en lien avec le texte littéraire étudié. La question de la méthode d'analyse et le choix des activités à mettre en place sont également fréquemment mentionnés.

5 Nous présentons ici les résultats d'une étude exploratoire menée à l'Inspé de MidiPyrénées, entre 2018 et 2020 et portant sur la séance de lecture de l'image au sein de la séquence de français. Ces résultats s'appuient sur l'analyse de deux éléments : d'une part les réponses à un questionnaire soumis à 50 professeurs stagiaires de lettres; et d'autre part, un échantillon représentatif de séances extraites d'un corpus d'une séquence de français, conçue par quatre de ces mêmes professeurs. Il leur a été également demandé d'accompagner leur séance de notes rendant compte des difficultés rencontrées.

6 Nous exposerons dans un premier temps les soubassements théoriques relatifs à la «lecture» de l'image, puis nous présenterons les choix méthodologiques que nous avons opérés, nous explorerons ensuite les déclarations concernant la place et le statut de l'image dans les séquences de lettres à partir du questionnaire et enfin analyserons plus précisément quatre séances de lecture de la première séquence de français au collège menées par quatre de ces professeurs stagiaires.

\section{Cadre théorique}

7 La « lecture » de l'image telle qu'elle est préconisée par les programmes de collège et de lycée conduit à se demander ce que signifie précisément « lire » une image et quelles compétences y sont convoquées.

8 Globalement, l'image peut se définir comme une composition d'éléments plastiques formant une interaction avec un médium. L'espace plastique (surface de l'image, organisation et composition, luminosité, gamme des couleurs, éléments graphiques simples, matière de l'image) est alors le point de contact « entre l'espace du spectateur et l'espace de l'image » (Aumont, 1990, p.101 sq.). À partir de cette définition de l'image, sa "lecture " peut-elle être envisagée autrement que comme la lecture d'un texte littéraire, sachant que la lecture sémiotique de l'image privilégie l'activité d'un spectateur guidé par les injonctions de celle- ci ? Cette lecture de type structuraliste développée par R. Barthes (1964) s'est appuyée sur un modèle unique qu'elle a cherché 
à étendre à tous les objets sémiotiques : pour lui, l'ordre du discours fournit des outils capables d'interpréter et d'analyser les autres modes d'expression. Dans le même esprit, E. Gombrich (1950) explique que la représentation picturale est la traduction du réel et il étend ainsi le concept de convention issu de la littérature à l'activité du peintre : styles et écoles sont affaire de codes culturels, et la sémiotique conduit à adopter les règles rhétoriques pour comprendre l'image. Celle-ci, pour reprendre R. Barthes (1964, p. 31), est tenue de passer par l'«ancrage", fonction du langage qui correspond au contrôle imposé par le texte à la polysémie de l'image ou au contraire par le « relais » qui appelle la complémentarité entre texte et image. La conception de R. Barthes ne prend cependant pas en compte la dialectique du rapport texte-image en termes de réception, donnant ainsi le primat au linguistique sur le perceptif (Joly, 2009, p. 25, sq.). Quant à J. Aumont (1990, p. 193), il précise que « pour être pleinement comprise, une image nécessite la maitrise du langage verbal ». L'image est traversée par le flux du discours et virtuellement porteuse d'un prolongement linguistique puisqu'elle est «rapportée par ses spectateurs historiques successifs à des énoncés idéologiques, culturels, en tous cas symboliques, sans lesquels elle n'a pas de sens » (ibid., p. 194). La question du sens de l'image est celle du rapport entre images et mots.

9 Cependant l'inféodation de l'image à la langue a été remise en question par les travaux du Groupe $\mu$ (1992) qui propose un modèle de signes iconiques propres à l'organisation des images. Dans son Traité du signe visuel, ce groupe, dans une perspective pragmatique, met en avant les propriétés spécifiques de l'image, caractérisée par des signes iconiques (lignes, traits, composition) et des signes plastiques (couleurs, textures, formes). Ainsi l'image est-elle constituée par l'agencement de ces signes, motivés par un contexte et interprétables. En partant des théories du groupe $\mu$ et en les relisant à la lumière de la sémiotique de C. S. Peirce, M. Joly (2009) reprend la théorie des trois catégories peirciennes du signe et les applique à l'image. Celle-ci peut donc être décomposée en indices, qui sont des traces directement prélevées du réel, en icônes, qui sont des figurations analogiques et en symboles, qui sont des représentations liées à des codes culturels. Lire une image consiste alors, en s'appuyant sur ses propriétés plastiques et graphiques, à repérer des signes interprétables et signifiants. D'autre part, J. Aumont a montré que l'image avait essentiellement trois fonctions :

- épistémique quand l'image apporte des informations sur le monde (carte à jouer ou carte routière par exemple ou encore illustrations de textes littéraires comme les manuscrits enluminés) ;

- symbolique quand elle représente des figures culturelles (colombe du Saint Esprit, représentation de Dieu en vieillard ou images allégoriques);

- esthétique enfin quand elle provoque des sensations spécifiques chez son spectateur.

Ces fonctions, selon lui, passent forcément par la verbalisation de leur réception par le spectateur, ce qui a conduit M. Picard (2002) à interroger l'activité artistique du point de vue de ce dernier qui déchiffre, lit, comprend ou pas, fantasme ou projette. Au final, plutôt que d'opposer lecture de l'image et lecture du texte, J. Rancière (2003) convie à déconstruire la séparation entre art des mots et art des formes, art du lisible et art du visible, et partant à repenser la construction de l'expérience sensible.

11 L'utilisation de l'image dans le cours de français pose question en particulier sur les outils d'analyse qui sont généralement empruntés à l'analyse littéraire (Demougin, 2003, 2005). L'image serait un équivalent iconique du texte littéraire, malentendu qui 
occasionne des simplifications de sa "lecture» et des transferts qui risquent de l'instrumentaliser et de l'affadir. De fait, l'image arrive toujours en second, comme complément au texte (Massol, Duvin-Parmentier \& Claude, 2020). De plus, l'approche sémiologique est valorisée alors que d'autres lectures, sociologiques, esthétiques ou psychanalytiques (Debray, 1992) pourraient revitaliser l'appréhension de l'image en évitant l'écueil d'un décryptage techniciste et en accordant une place à l'expression des émotions, des sentiments ou des jugements du spectateur.

12 Le groupe Sémiotiques de l'INRP (1983), en lien avec les travaux structuralistes de R. Barthes dans les années quatre-vingt, pose les bases d'une recherche sur la place de l'image dans le cours de français et analyse plus précisément la fonction heuristique de l'image pour travailler la langue. Cependant les interactions didactiques entre français et arts n'apparaissent que bien plus récemment. Ainsi pour J.-L. Dufays, L. Gemenne et D. Ledur (2005), l'image peut faire l'objet d'une appropriation comparable à la lecture littéraire, physique, imaginaire et intellective suivant les catégories de M. Picard (1986). Le va-et-vient entre texte et image suscite alors des "attitudes participatives et critiques, entre des jugements d'ordre éthique, esthétique, référentiel ou autre " (Dufays, Gemenne \& Ledur, 2005, p. 314).

L'introduction de l'histoire des arts en 2008 a conduit à réévaluer la question du champ disciplinaire (Duvin-Parmentier, 2010) et redonner de la légitimité à la lecture de l'image en français, comme en témoigne le numéro 182 de la revue Le Français aujourd'hui : « Histoire des arts : de la notion à la discipline » (Baldner \& Barbaza, 2013), qui postule que l'écriture sur l'image contribue à former et à approfondir le jugement esthétique et critique tout en conduisant à l'élaboration du rapport au monde d'un spectateur émancipé. Le numéro 175-176 (Leclaire-Halté \& Petijean, 2017) de la revue Pratiques intitulé "Pour un dialogue interdidactique français/art» fait état d'une nécessaire didactisation du lien entre lecture de l'image et lecture du texte littéraire. J.C. Chabanne $(2013,2017)$ rappelle l'intérêt pour la didactique du français de dialoguer avec les didactiques des arts. Selon lui, ces disciplines s'enrichissent mutuellement et le discours tenu sur l'art ne relève pas uniquement de l'utilisation d'un lexique et de techniques spécifiques mais de formes discursives subjectivantes et singulières que la littérature suscite. La polysémie du langage est donc indissociable des approches de l'œuvre d'art. Ainsi, « lire » l'image conduit à convoquer les richesses composites du langage, à mêler paroles expertes et expressions du ressenti sensible, à développer la capacité à parler de l'art et sur l'art. La question centrale porte alors sur ce qui est en jeu dans la « lecture » de l'image en lien avec le texte littéraire : si l'image conduit au texte par le biais d'une multitude discursive, elle ne se confond toutefois pas avec celuici et reste une expérience singulière de réception. Pour F. Demougin $(2003 ; 2005)$ comme pour J.-C. Chabanne, la posture de l'enseignant de français est destinée à évoluer parce qu'il est nécessaire d'introduire un mode de lecture rendant compte d'un parcours de l'élève, personnel et engagé, par le regard et par la parole. J.-C. Chabanne et M.-S. Claude (2016) préconisent un tissage entre expression du sensible et connaissances intellectives d'autant que les enseignants débutants sont souvent prisonniers de leur formation universitaire et ont du mal à exprimer leur propre approche personnelle et sensible de l'œuvre (Deronne, 2011). Pour en finir avec «le décodage rationnalisant " (Picard, 1986) et les dérives formalistes et technicistes, la lecture de l'image doit s'ouvrir à la subjectivité et aux émotions de lecteurs singuliers, à l'instar de la lecture littéraire. Enfin, la question de la représentation de l'image dans la séquence de français chez les professeurs a été étudiée par M.-S. Claude (2014) qui a 
confronté les discours de la recherche et ceux des professeurs. M.- S. Claude pose la question centrale du détour par le commentaire pictural pour aborder le commentaire littéraire et s'interroge sur les représentations que les enseignants ont de l'image. Or elle constate que ces derniers sous-estiment la nécessité d'expliciter, tant les points de convergence entre peinture et littérature que l'intérêt du détour par l'image. En formation initiale, il semble donc nécessaire que les professeurs débutants puissent se situer par rapport à ces représentations afin de lever certains malentendus dans l'exploitation de l'image.

\section{Méthodologie de la recherche}

14 Notre objectif visait à mieux cerner les représentations qu'ont les enseignants débutants de la place de l'image dans la séquence de français et la façon dont ils s'emparent ou pas des réactions singulières $d u$ "spectateur empirique » face aux sollicitations de l'œuvre. Nous nous sommes également intéressées aux difficultés rencontrées par eux dans la préparation de leur séance et/ou lors de la séance ellemême.

15 À cet effet, nous avons soumis dans un premier temps, entre 2018 et 2020, un questionnaire à 50 professeurs stagiaires de l'Inspé de Midi-Pyrénées. Rappelons que, comme pour tout questionnaire, il n'est pas toujours possible de faire émerger les représentations mentales, les opinions et les perceptions des participants (Wirthner \& Garcia-Debanc, 2010). Nous avons ensuite mis en regard les réponses au questionnaire avec un corpus de séances de lecture de l'image. Ces séances avaient été menées par les mêmes professeurs en début d'année scolaire. Nous présenterons les résultats du questionnaire ainsi qu'un échantillon de 4 séances.

Afin d'établir un premier état des lieux, et en préambule à la constitution d'un corpus plus conséquent, nous avons privilégié les questions ouvertes.

Les questions posées étaient les suivantes :

- Intégrez-vous une image dans votre séquence de français? Pourquoi?

- Pouvez-vous préciser à quel moment?

- Comment choisissez-vous cette image?

- Utilisez-vous une méthode particulière pour analyser une image?

- Quelles difficultés rencontrez-vous lors de la préparation de votre séance?

- Quelles difficultés rencontrez-vous lors de la lecture de l'image en classe?

\section{Résultats de la recherche (1) : ce que nous apprend le questionnaire}

\section{Une utilisation polyvalente de l'image}

Le premier constat est l'utilisation massive de l'image (91\%). Les enseignants notent que l'attirance pour l'image fédère la classe et entraine une prise de parole $(78,5 \%)$. Cette mise en appétence vient de ce que l'image peut être par exemple perçue comme une énigme à résoudre $(32,5 \%):$ «Je l'utilise en début de séquence. Comme ils adorent travailler l'image, cela me permet de leur faire deviner la séquence et on crée déjà une attente par rapport aux textes ». Le pouvoir rhétorique de l'image, la captation et 
l'adhésion qu'elle suscite chez les élèves contribue à construire des attentes de lecteur (73\%). Ainsi, l'analyse péritextuelle des couvertures de livres prépare l'entrée dans la lecture : «Je l'utilise pour soulever des hypothèses. Avec les sixièmes j'ai pu aborder Les Fourberies de Scapin. J'ai projeté plusieurs couvertures et ils devaient faire des hypothèses sur les personnages, le lieu où se déroule la pièce. "

L'image favorise la compréhension des textes $(69,5 \%$ des cas) en donnant à voir descriptions et personnages (38,5\%). Pour la majorité des professeurs, l'image vient en auxiliaire avec pour finalité de faciliter l'entrée dans la lecture des textes littéraires, jugée plus complexe: "Je me sers de l'image parce qu'elle me semble plus accessible pour entrer dans des textes littéraires parfois complexes ». Un professeur précise que le détour par l'image (M.-S. Claude, 2014) peut être bénéfique pour les élèves les plus fragiles : «Les arts visuels sont plus accessibles que la littérature. Cela permet aux plus faibles de comprendre certains mouvements littéraires ». Cette représentation de l'image d'accès plus facile que le texte ne prend toutefois pas en compte la complexité du lien entre texte et image. De fait, la fonction essentiellement descriptive de l'image est souvent mentionnée : «Ma première séquence portait sur la ville, j'avais choisi une photographie de Abbott (Nightview of New York) et Levitt (New York) pour illustrer un poème de Cendrars sur la ville, Pâques à New York. ", fonction des plus explicites selon $\mathrm{J}$. Aumont pour qui la représentation de l'image comme une analogie au texte vient de sa conception essentiellement illustrative.

L'image contribue également à éclairer des notions complexes (13,5\%): «Pour travailler la notion d'atmosphère propre à la poésie romantique, le tableau de C. D. Friedrich a permis aux élèves de s'appuyer sur des représentations visuelles ». L'image apparait alors comme un outil polyvalent à même d'éclairer des aspects très divers du texte littéraire (sa thématique, son esthétique, sa stylistique...). La séquence consacrée à la poésie romantique par exemple est illustrée par Le Voyageur contemplant une mer de nuages (1818) de C.D. Friedrich, une œuvre contemporaine, avec une thématique correspondant à celle des textes et une esthétique romantique : «J'insiste sur le lien avec ce que l'on est en train d'étudier ; j'insiste sur le sens de cette activité : en quoi elle peut enrichir le propos, ce qu'elle apporte, autant par son esthétisme que par le message transmis ». La question du lexique en lien avec le texte est peu convoquée. L'utilisation d'un lexique technique de l'analyse de l'image (10,5\%) semble recevoir peu d'écho chez les professeurs, sans doute parce que ces derniers disent ne pas viser une lecture de spécialiste: "J'utilise avec eux quelques mots clés comme composition ou couleur mais pas davantage ».

Tableau 1. Image et parcours de lecture

\begin{tabular}{|l|c|}
\hline Faciliter la compréhension du texte (support pédagogique) & $69,5 \%$ \\
\hline Favoriser des attentes de lecture & $41 \%$ \\
\hline Image descriptive (thématique, personnages, lieux) en lien avec le texte & $38,5 \%$ \\
\hline Entrer dans l'atmosphère du texte & $13,5 \%$ \\
\hline $\begin{array}{l}\text { Donner ses impressions et utiliser un lexique adapté en lien avec le texte : la réception de } \\
\text { l'image }\end{array}$ & $13,5 \%$ \\
\hline Analyser une image et utiliser un lexique adapté en lien avec le texte & $10,5 \%$ \\
\hline
\end{tabular}


$\mathrm{Au}$ final, ces réponses indiquent que la posture dominante d'une lecture sémiotique experte et distanciée prévaut sur l'expression de la subjectivité. En effet dans seulement 13,5\% des cas, la construction de l'interprétation met en avant l'implication de l'élève et la verbalisation d'un parcours personnel : «[L'image] conduit les élèves à exprimer leurs avis sur le tableau alors que certains sont moins à l'aise avec la lecture textuelle, ils osent prendre la parole et sont étonnés d'avoir beaucoup à dire ». Le lexique de la réception et de l'expression des émotions n'est pas non plus souvent mentionné $(10,5 \%)$, sans que cela signifie pour autant que le rapport singulier et subjectif à l'image ne soit pas pris en compte mais plutôt qu'il ne fait pas l'objet d'un travail sur la langue.

\section{Une lecture préambule}

In fine, l'idée d'une image redondante avec le texte est donc nuancée puisque la variété des réponses développées dans le questionnaire montre que l'image prépare et cristallise différents parcours de lectures du texte littéraire.

La conséquence de ces représentations du rôle de l'image dans la séquence de français conduit à l'introduire souvent dans la première séance, en préambule à l'entrée dans les textes de la séquence (79\%). Elle sollicite alors diverses postures de lecture et en particulier ouvre des horizons d'attente sur les textes (41\%). Une fois l'image analysée, peu de retours sont faits : "Généralement je ne fais plus référence au tableau étudié en début de séquence » ou "Les élèves font parfois référence au tableau quand on étudie un texte mais c'est rare ». Cependant, dans $13 \%$ des cas, l'image est donnée en fin de séquence pour enrichir le texte étudié. Elle sert aussi à vérifier la compréhension et l'interprétation du texte. Dans les deux cas, cette mise en silence de l'image, qui correspond au recentrement de la parole sur le texte, ne conduit pas forcément à une véritable dialectique dans le cours de la séquence, et la question de la transversalité de la langue qui traverserait le champ artistique (Chabanne, 2016) reste un point aveugle.

\section{Le choix des méthodes de lecture de l'image : une approche essentiellement intellective}

Dans la première partie du questionnaire, l'image a été présentée comme un outil facilitant l'entrée dans le texte littéraire mais le manque de méthode, dans $84 \%$ des cas, et le manque de culture artistique, dans $68 \%$ des cas, rendent l'exploitation de l'image problématique. Cependant, il faut largement tempérer ce point dans la mesure où les professeurs débutants semblent, tout en déplorant la difficulté à mettre en place une analyse de l'image, se servir en réalité de méthodes éprouvées.

Tableau 2. Méthodes d'analyse de l'image

\begin{tabular}{|l|l|}
\hline Méthode iconographique (description, analyse, interprétation) & $71,5 \%$ \\
\hline Verbaliser une expérience sensible & $31 \%$ \\
\hline Méthode comparatiste (comparaison thématique, stylistique... image/texte) & $14 \%$ \\
\hline Partir d'un détail pour analyser l'ensemble & $4 \%$ \\
\hline
\end{tabular}




\section{l'image relève principalement de la tradition iconographique (71,5\%), sans doute parce} que c'est ce qui demeure largement diffusé dans les programmes de français et dans ceux d'histoire des arts (2008) quand eux-mêmes étaient élèves. Cette lecture iconographique s'articule en trois étapes inspirées de la méthode d'E. Panofsky (1969) et influencées par la lecture sémiotique de R. Barthes : lors du stade pré-iconique, le spectateur, en posture d'observateur, décrit l'image ; au stade iconique, il est conduit à faire des inférences et rattacher l'œuvre à un motif conventionnel; au stade iconologique, il met au jour les «significations intrinsèques" de l'image en la confrontant à d'autres œuvres picturales, littéraires, ou à des documents divers (culturels, politiques, économiques, historiques) qui viendront enrichir son analyse. La posture de spectateur se construit ainsi suivant un déroulement déterminé. Cette méthode nécessite un étayage important de la part de l'enseignant, comme le confirme un professeur : "Il a fallu que je leur montre où diriger leurs yeux, quels termes utiliser ». Au stade iconologique, l'interprétation des élèves est source d'approximations et de contresens. Une enseignante souligne à ce propos le décryptage des symboles et la contextualisation comme les deux obstacles à la compréhension de l'œuvre: "Je les guide surtout pour éviter qu'ils fassent des contresens sur les symboles et aussi sur les anachronismes car il est difficile pour eux de se rendre compte de leur signification véritable ».

D'autres approches sont mentionnées, par exemple, quand l'expression d'une expérience sensible $(31 \%)$ sert d'ouverture à la séance : « Pour le tableau de Friedrich, nous sommes partis de leurs impressions, je trouvais que c'était plus pertinent pour entrer dans l'intime en poésie. "

Une autre méthode, comparatiste $(14 \%)$, consiste à mettre en regard des images principalement regroupées par thème et par époque : «J'ai présenté des tableaux que nous avons analysés oralement. Nous les avons comparés à des peintures d'histoire du $\mathrm{XIX}^{\mathrm{e}}$ siècle car je souhaitais que les élèves comprennent que dans la peinture réaliste, il y a une absence d'événements et de personnages historiques ». Cette méthode parait pertinente aux professeurs qui veulent construire un parcours historique : «J'aime bien donner une série d'images, je leur demande de les classer par ordre chronologique pour qu'ils voient l'évolution artistique ». La lecture comparée de plusieurs images conduit ainsi à dégager des "patrons" iconiques autour de thématiques communes comme le fait remarquer ce professeur: "Je regroupe plusieurs tableaux pour que les élèves voient les points communs, cela nous permet de constituer des grilles de lecture ». Une dernière méthode, peu mentionnée (4\%), vise, à partir d'un détail dans le tableau, à tirer le regard d'un point signifiant vers l'ensemble de l'image. Ainsi ce professeur qui, à l'occasion d'une séquence sur le fantastique, invite ses élèves à considérer plus particulièrement deux éléments symboliques du tableau de J.-H. Füssli Le Cauchemar (1781) - en l'occurrence un succube couché sur le corps d'une femme et une tête de cheval au second plan - pour opérer dans un second temps une lecture globale : "Cela permet de comprendre que la femme n'est pas morte mais rêve. Partir de ces deux éléments conduit à regarder autrement l'ensemble. »

Donc les méthodes sont relativement variées et, même si la lecture iconographique est dominante, les professeurs s'essaient à d'autres parcours de lecture, ce qui laisse supposer chez eux des compétences plus importantes que ce qu'ils affirment. 


\section{Les difficultés dans les déclarations des professeurs}

Tableau 3. Les difficultés rencontrées

\begin{tabular}{|l|l|}
\hline Manque de méthode dans la préparation de la séance & $84 \%$ \\
\hline Manque de connaissances artistiques des professeurs & $74 \%$ \\
\hline Manque de formation des professeurs & $59 \%$ \\
\hline Manque de temps en classe & $80,5 \%$ \\
\hline Manque de culture des élèves & $53,5 \%$ \\
\hline
\end{tabular}

Les difficultés déclarées face à la lecture de l'image apparaissent en même temps que d'autres, liées davantage au contexte de l'exercice en classe, celles, précédemment mentionnées sur le manque de méthode (84\%), s'accompagnant par exemple d'un défaut de culture artistique (74\%). La gestion du temps $(80,5 \%)$ conduit également à un sentiment d'insatisfaction. Un professeur explique qu'une lecture trop dirigée ne permet pas d'instaurer un véritable dialogue avec la classe: «C'est assez frustrant, comme on manque de temps, je les guide sans arrêt, en introduisant des éléments théoriques tels que les plans, la perspective, les contrastes et il faut faire surgir des remarques et imposer des choix ». La réception de l'œuvre, pour cette même raison, n'est pas toujours prise en compte, comme le déplore cette enseignante : «Il faut aussi ensuite les questionner sur leur ressenti, mais je n'ai pas toujours le temps de le faire ». En revanche, le manque de culture artistique chez les élèves n'est évoqué qu'à 53,5\% par les professeurs qui précisent qu'ils ne parviennent pas à pallier cette méconnaissance : "L'histoire des arts est un long processus d'apprentissage que j'avais sous-estimé ». Les élèves restent le plus souvent bloqués au stade pré-iconique et rencontrent des difficultés au stade iconique.

Enfin, la promesse d'un plaisir esthétique qui pourrait découler de l'utilisation de l'image ne se réalise pas forcément selon ce même professeur: "Alors que je pensais naïvement que la fréquentation des œuvres d'art suffirait à ce qu'ils se rendent compte de ce qui est beau, je me suis aperçue très vite que les codes du beau en art sont régis par des codes culturels compris par des initiés. »

\section{Les activités autour de la lecture de l'image}

Tableau 4. Exploitation des activités liées à l'image dans la séquence

\begin{tabular}{|l|l|}
\hline $\begin{array}{l}\text { Une activité orale d'analyse de l'image avec éventuellement trace écrite (résumés, } \\
\text { définitions) }\end{array}$ & $81 \%$ \\
\hline Écriture descriptive (stade iconique) & $18,5 \%$ \\
\hline Écriture narrative & $13 \%$ \\
\hline Écriture de la réception & $5,5 \%$ \\
\hline
\end{tabular}


activités générées par la lecture de l'image sont essentiellement orales (81\%) et peuvent être accompagnées d'une trace écrite, généralement un résumé de ce qui a été dit collectivement. Les autres écrits sont peu fréquents: sont cités les écritures narrative $(18,5 \%)$ et descriptive $(13 \%)$ ou encore les écrits de la réception (5,5\%) qui rendent compte d'une lecture subjective dans une rencontre personnelle avec l'image : «Les œuvres sont toujours utilisées en partant des impressions ressenties des élèves puis je procède à une analyse formelle de ce que l'on voit, je le fais uniquement à l'oral, jamais à l'écrit parce que je veux entrer dans l'analyse du texte ». Notons toutefois que les écrits de réception proprement dits ne semblent pas faire l'objet d'une exploitation particulière comme l'indique un professeur : "Les élèves sont amenés à donner leurs impressions sur l'œuvre et à écrire quelques phrases au brouillon mais jamais plus de cinq minutes. Puis la mise en commun permet de voir les différentes perceptions. Je préfère qu'ils expriment rapidement leur ressenti avant d'entrer dans l'analyse ». Ainsi, au cours de la séance, l'expérience esthétique cède-t-elle la place à l'analyse, et le va-etvient entre émotion et cognition reste un point aveugle de ces réponses.

Ce questionnaire n'est évidemment pas suffisant pour rendre compte de la représentation de la place de l'image dans l'approche de la lecture littéraire, ni des savoirs et des savoir-faire mis en œuvre par les professeurs dans la préparation de leur séance de lecture de l'image. Il ne renseigne pas davantage les savoirs en acte lors de la séance en classe.

'affiner notre approche, nous avons donc considéré la préparation de la première séquence de l'année; nous avons examiné la préparation de la séance de lecture de l'image ainsi que les annotations que nous avions demandées concernant les difficultés rencontrées.

\section{Résultats du recueil de données (2) : la préparation des séances de lecture de l'image}

Le corpus de séances de lecture de l'image a été alimenté par les séquences des professeurs stagiaires qui ont répondu au questionnaire. Nous avons ainsi analysé 48 préparations de séances annotées par les professeurs après leur exploitation en classe, en ayant pris le parti de nous limiter à la première séquence de l'année, avant le début du module optionnel de formation en histoire des arts.

le cadre de cet article, nous avons choisi d'analyser quatre séances menées en collège lors de la première séquence de l'année. Les quatre professeurs retenus sont ceux dont nous assurons le suivi en tutorat, ce qui nous permet de mesurer de façon longitudinale les effets produits par la formation. Avant l'exploitation de leurs séances, un bref entretien oral a permis de brosser un portrait succinct des enseignants, en nous intéressant plus particulièrement à leur parcours universitaire (formation en histoire de l'art ou en histoire des arts) et à leur rapport à l'art (fréquentation des musées ou galeries, lecture d'ouvrages d'art...). Les quatre séances ont été retenues car elles illustrent la variété des images retenues et leur pertinence au niveau thématique, historique, esthétique ou symbolique dans leur lien avec le texte littéraire.

Nous allons commencer par une rapide présentation des professeurs. Pierre note ne pas avoir de connaissances spécialisées en art mais apprécier la peinture, fréquentant occasionnellement les musées (deux à trois fois par an), surtout lors de voyages.

Pratiques, $187-188 \mid 2020$ 
Daphnée, qui explique qu'elle pense ne pas apprécier la peinture à sa juste valeur, souhaite se former parce qu'elle estime que cela compléterait sa formation littéraire. Julien se dit attiré parl'art en général et l'image en particulier, qu'il intègre systématiquement dans ses pratiques. Quant à Laurence, elle a suivi une unité d'enseignement en histoire de l'art durant sa formation universitaire et estime qu'il est nécessaire de sensibiliser ses élèves à l'art, d'autant, précise-t-elle, qu'ils « sont en REP+ et géographiquement éloignés des centres artistiques. »

\section{Choix des images et place de la séance de lecture de l'image dans la séquence}

Pierre collecte des images au cours de ses visites dans les musées et de ses rencontres avec l'art, celles qu'il utilise faisant référence au parcours d'un spectateur singulier: "J'ai mon petit musée personnel, un classeur dans lequel je conserve des images qui vont me servir en cours ». Il dit récolter des images plus rarement montrées : «J'ai ainsi une grande latitude dans le choix; je n'ai pas toujours envie de me servir des images qu'on trouve dans tous les manuels. J'ai envie de montrer autre chose ». Daphnée choisit ses œuvres en fonction d'objectifs littéraires déterminés: "Il m'arrive de donner des toiles que je n'apprécie pas (par exemple pour le Réalisme) car elles me semblent importantes du point de vue des jalons culturels et de la compréhension des textes ». Quant à Julien, il fait confiance à son goût personnel pour choisir ses images : «Ma sensibilité, c'est ce qui me guide, j'aime donner des œuvres (littéraires comme picturales) que j'aime ». Laurence enfin intègre systématiquement une image, voire plusieurs, et ce, à différents moments de la séquence selon les objectifs qu'elle poursuit, à la fois comme séance d'accroche ou comme embrayeurs d'écriture pour les sujets de rédaction et les ateliers écriture en classe. L'image peut avoir en outre une fonction conclusive : "Il m'est arrivé aussi d'y consacrer ma toute dernière séance comme en séquence 1 où je trouvais pertinent de voir l'évolution du réalisme pictural comme aboutissement ». Chez Laurence, en plus du rôle de catalyseur de l'image, que nous avons déjà rencontré dans le questionnaire, une dialectique entre lecture littéraire et picturale, entre lisible et visible peut donc apparaitre en fin de séquence. Elle explique enfin vouloir que les élèves partagent leurs impressions dans le but de créer un espace intersubjectif commun: "J'avais pu relever que les activités que j'avais bâties sur l'appel à l'intuition des élèves fonctionnaient bien, notamment mes différentes activités consistant à leur faire dégager des impressions. »

Les quatre enseignants apportent des réponses contrastées qui ne correspondent pas toujours avec les réponses au questionnaire. Daphnée et Laurence opèrent un choix pragmatique dans lequel leurs goûts personnels entrent peu ou pas en ligne de compte tandis que Pierre, en constituant son musée imaginaire, collecte des œuvres qui lui plaisent ou dont il pressent l'intérêt pour une exploitation en classe, et que Julien s'appuie aussi sur ses émotions et sa sensibilité. Seule Laurence pourtant prend en compte la réception de l'image chez ses élèves qu'elle intègre à ses objectifs de lecture.

\section{Présentation de la séance de la première séquence} débute par la projection d'un corpus de tableaux réalistes (Courbet, Degas, Caillebotte, 
Manet \& Monet) représentant des scènes de travail. Ses questions portent sur la thématique commune des textes et des images ainsi que leur appartenance au courant réaliste. L'enseignant fait état de la motivation des élèves; il regrette cependant que l'image n'ait ici qu'une fonction de validation de la lecture des textes: «Je n'ai pas réussi à leur faire regarder l'image en tant que telle, ils étaient uniquement dans la comparaison avec les textes et je pense qu'au final j'ai instrumentalisé les tableaux. »

La séquence de Daphnée porte sur la figure du monstre en $6^{\mathrm{e}}$. C'est la première séance qui est consacrée à la lecture de l'image, en l'occurrence une peinture sur bois de Kunisado Ukagawa (1859) représentant une vieille femme aux allures de sorcière. La séance s'ouvre sur un questionnement quant aux représentations de la sorcière chez les élèves puis l'image est projetée et, lors d'un oral collectif, certains de ses éléments caractéristiques sont relevés (thématique, composition et couleurs). Les élèves doivent ensuite en proposer un titre puis imaginer l'objet de la séquence. Les commentaires de Daphnée insistent sur la motivation des élèves : "La thématique leur plait et ils ont beaucoup aimé l'image ». Cependant elle regrette d'avoir négligé la prise en compte de la réception de l'image: "Je n'ai pas su exploiter leurs remarques plus personnelles; j'aurais dû travailler sur leur sentiment de peur, cela m'aurait permis d'aller plus loin dans l'analyse des textes ". Lors des échanges oraux, Daphnée régule les réponses dans la perspective de la future entrée dans les textes: «Je saisis les remarques des élèves utiles pour les textes et je mets en valeur leurs analyses lorsqu'elles sont intéressantes. Parfois nous notons des éléments d'analyse dans le cahier». Globalement, elle se montre satisfaite de cette séance, qui a permis d'éveiller des attentes de lecture des textes : « Ils aiment les histoires de monstre et l'image a bien fonctionné dans ce sens. »

41 Julien, dans une séquence en $5^{\mathrm{e}}$ intitulée « Regarder des mondes, inventer des mondes, imaginer des univers nouveaux ", a consacré la séance inaugurale à un tableau de R. Gonsalves L'Espace entre les mots. Son objectif consiste à introduire une analyse comparée entre cette image et un extrait d'Alice au pays des merveilles de L. Carroll, et cela à partir du topos de la porte ouverte comme passerelle vers un monde imaginaire. La séance débute par une présentation du réalisme magique et du travail de $R$. Gonsalves. Le tableau est ensuite projeté et l'attention des élèves est guidée sur les portes, détail qui cristallise l'intrusion du magique dans une scène réaliste : «Je voulais montrer qu'une image peut se lire comme un texte, elle raconte des histoires et le motif de la porte me semblait pertinent parce qu'on le retrouve dans le tableau et dans Alice ». Julien constate cependant n'avoir pas atteint l'objectif qu'il s'était fixé parce que les lectures de l'image et du texte ont été menées séparément : « Mon analyse comparée n'en était pas vraiment une, j'ai essayé de faire des liens entre le motif de la porte (terrier du lapin dans le texte et hublot dans l'image) mais je n'ai jamais confronté les deux supports ». De fait, le choix de l'image manque de pertinence puisque le motif de la porte dans l'image n'y occupe pas la même fonction symbolique que dans le texte : en effet, chez R. Gonsalves, la démultiplication angoissante des portes ouvertes sur la mer, telle une impasse, donne une impression d'enfermement tandis que chez L. Carroll, l'entrée du terrier du lapin est une invite à la découverte d'un nouveau monde.

Chez Laurence, la lecture de l'image ouvre une séquence consacrée à la nouvelle fantastique en $4^{\text {e }}$. Une courte production écrite en début de séance vise à repérer l'atmosphère fantastique et la présence d'éléments étranges dans l'image. La consigne : "Rédige un paragraphe sur tes impressions sur le tableau de Friedrich Un moine dans la neige et dresse une liste de 5 mots clés qui te viennent à l'esprit en regardant ces 
tableaux » met en lien expression des émotions et lexique. Les élèves lisent ensuite leur texte à la classe et l'enseignante note au tableau le lexique récurrent ("bizarre", " angoissant ", « lugubre », « étrange »...) : ce partage du sensible peut être interprété comme un moment intersubjectif. Elle projette ensuite Le Cauchemar de J.-H. Füssli pour qu'à partir des éléments communs avec le tableau de Friedrich, soit dégagée une définition du fantastique. Enfin, Laurence note que l'expression du sensible aurait pu être reprise en fin de séance dans un va-et-vient dynamique entre lecture personnelle et lecture intellective: "J'aurais pu les réinterroger sur leurs impressions après l'analyse, souvent ils n'aiment pas un texte au début et une fois qu'on l'a travaillé, ils me disent qu'ils le trouvent beau. »

\section{Difficultés dans la préparation et dans la mise en œuvre}

43 Pierre souligne qu'il ne parvient pas à clairement définir le statut de l'image, qu'il exploite plutôt comme une illustration: "Je crois que j'instrumentalise l'image; je n'arrive pas à la penser en tant que telle mais comme un moyen de faciliter l'entrée dans la lecture ». Daphnée se heurte à un autre type d'obstacle: en se voulant exhaustive, elle perd de vue la finalité de l'image et ne sait pas réellement créer un lien entre celle-ci et le texte : "J'ai l'impression de ne pas assez maitriser la question pour déterminer les points pertinents à travailler dans l'image, je finis par ne plus trop voir le rapport entre l'image et le texte ». Julien a le même type de problème au niveau de l'articulation entre les deux sémiotiques et donc avec la prise en compte de la spécificité du langage de l'image: «J'ai du mal à analyser l'image autrement que comme un texte; mes questionnements sont les mêmes alors que les élèves ne les mettent pas sur le même plan ». Laurence ne rencontre pas de difficultés particulières pour préparer sa séance : sa connaissance de l'histoire de l'art l'a conduite à suivre une tradition iconologique et elle possède les outils sémiotiques propres à la lecture de l'image. Toutefois elle peine dans la transposition didactique de ses connaissances et souligne qu'elle est souvent étonnée par les questions et les difficultés des élèves qu'elle ne parvient pas toujours à anticiper : "Je suis surprise quand je vois qu'ils ne savent pas du tout situer une œuvre dans son époque. Pour eux, L. de Vinci, c'est le XIX ${ }^{\mathrm{e}}$ siècle. »

Toutefois, si pour chacun des professeurs stagiaires utiliser une image semble aller de soi, on peut s'interroger sur leurs choix didactiques quant au statut qu'ils donnent à celle-ci, notamment concernant la (re)connaissance ou non de sa spécificité sémiotique. Dans les commentaires de ces professeurs transparait d'ailleurs une frustration qui vient de leur recours quasi systémique à des pratiques littéraires pour s'approprier l'image et donc au peu de place accordée à sa lecture subjective, au profit d'une lecture plutôt analytique.

\section{Conclusion}

Cet article cherchait à documenter les représentations des professeurs stagiaires de lettres, à travers leurs choix méthodologiques concernant la lecture d'image en lien avec le texte littéraire, ainsi que le type de difficultés rencontrées. Les résultats de notre étude exploratoire confirment l'évidente fonction motivationnelle de l'image qui se traduit par la participation effective des élèves. Il ressort également que l'image, 
lorsqu'elle est étudiée en première séance, induit des parcours de lecture divers, par le biais d'une thématique, d'une stylistique ou d'une atmosphère en lien avec le texte tandis qu'en milieu ou fin de séquence, elle valide la lecture littéraire. Cependant, dans la mesure où, convoquée comme auxiliaire, elle reste inféodée au texte, sa lecture, même si les dispositifs mis en œuvre avec les élèves sont variés, reste en partie académique et privilégie l'approche intellective et l'analyse. La grande majorité des professeurs mettent en cause leur manque de culture autant (voire davantage) que celui de leurs élèves. On peut aussi supposer que ces professeurs, en laissant de côté leur expérience personnelle et esthétique face à l'œuvre, induisent en classe ce type de lecture de l'image dans laquelle les activités autour de la réception sont peu exploitées.

La finalité de la formation des professeurs débutants à la lecture de l'image est à notre sens moins un élargissement des savoirs et des connaissances artistiques qu'une réévaluation de ce que signifie regarder une image de façon personnelle afin de mettre en place une dialectique dynamique entre expression du sensible et lecture experte, à l'instar de ce que la recherche didactique encourage pour la lecture littéraire.

\section{BIBLIOGRAPHIE}

AUMONT, J. (1990). L'Image. Paris : Nathan.

BALDNER, J.M. \& BARBAZA, A. (2013). « Histoire des arts : de la notion à la discipline ». Le Français aujourd'hui 182. Paris : A. Colin. En ligne : https://www.cairn.info/revue-le-francais-aujourdhui-2013-3-page-3.htm.

BARTHES, R. (1964). « Rhétorique de l'image ». Communication 4, p. 41-42. En ligne : https:// www.persee.fr/doc/comm_0588-8018_1964_num_4_1_1027.

CHABANNE, J.-C. (2013). « Histoire des arts et jeux de langage : comment parler de l'œuvre? ». Le Français aujourd'hui 182, p. 55-66. En ligne : https://www.cairn.info/revue-le-francais-aujourdhui-2013-3-page-55.htm.

CHABANNE, J.-C. (2016). « Approches interdisciplinaires des enseignements culturels et artistiques dans le cadre de l'enseignement de l'histoire des arts : un modèle complexe, une didactique impossible ?». Tréma. Revue internationale en sciences de l'éducation et didactique 45, p. 59-69. En ligne : https://journals.openedition.org/trema/3501.

CHABANNE, J.-C. (2017). «L'espace interdisciplinaire entre la discipline « français » et l'Éducation artistique et culturelle ». Recherches. Revue de didactique et de pédagogie du français 67, p. 63-92. En ligne : https://revue-recherches.fr/wp-content/uploads/2019/12/063-092_R97_Chabanne.pdf. CLAUDE, M.-S. (2014). « Commenter la peinture et la littérature : quels apports de la recherche pour la formation des enseignants? ». Recherche \& formation 77, p. 103-115. En ligne : https:// doi.org/10.4000/rechercheformation.2330.

CLAUDE, M.-S. (2016). « Lire la peinture, lire le texte littéraire à l'école : une activité de même nature ?».Éducation \& didactique 10 (1). En ligne : https://journals.openedition.org/ educationdidactique/2468. 
DEBRAY, R. (1992). Vie et mort de l'image. Une histoire du regard en Occident. Paris : Gallimard.

DEMOUGin, F. (2003). Voir ou lire. Pour une éducation du regard. Paris : L'Harmattan.

DEMOUGIN, F. (2005). «Littérature et formation du lecteur : la dynamique de l'image dans la construction du sujet ». Tréma. Revue internationale en sciences de l'éducation et didactique 24 , p. 27-35. En ligne : https://doi.org/10.4000/trema.664.

DERONNE, C. (2011). « Former des enseignants à exprimer leur expérience singulière d'une œuvre littéraire ou plastique ». Repères. Recherche en didactique du français langue maternelle 43, p. 103-126. En ligne : https://doi.org/10.4000/reperes.222.

DUFAYS, J.-L., GEMENNE, L. \& LEDUR, D. (2005). Pour une lecture littéraire. Histoire, théories, pistes pour la classe. Bruxelles : De Boeck.

DUVIN-PARMENTIER, B. (2010). Pour enseigner l'histoire des arts, regards interdisciplinaires. Amiens/ Paris : Scérén-CRDP/Crap-Cahier pédagogique.

GABATHULER, C. (2013). « Les arts visuels au secours de l'étrangeté littéraire. Analyse comparée de pratiques effectives sur un texte "réticent" ». La Lettre de l'AIRDF 53, p. 31-35. En ligne : https:// www.persee.fr/doc/airdf_17767784_2013_num_53_1_1959.

GABATHULER, C. (2016). Apprécier la littérature. La relation esthétique dans l'enseignement de la lecture de textes littéraires. Rennes : Presses universitaires de Rennes.

Groupe $\mu$ (1992). Traité du signe visuel. Pour une rhétorique de l'image. Paris : Editions du Seuil.

JOLY, M. (2009) [1993]. Introduction à l'analyse de l'image. Paris : A. Colin.

LeClaire-Halté, A. \& PetijeAn, A. (2017). « Pour un dialogue interdidactique français/arts ». Pratiques 175-176. En ligne : https://journals.openedition.org/pratiques/3553.

MASSOL, J.-F., DUVIN-PARMENTIER, B. \& CLAUDE, M.-S. (2020). « Image et arts visuels ». In : BrillantRannou, N., et al. (dirs). Un dictionnaire de didactique de la littérature. Paris : H. Champion, p. 141-147.

PANOFSKY, E. (1969). L'Euvre d'art et ses significations. Essais sur les « arts visuels ». Paris : Gallimard. PICARD, M. (1986). La Lecture comme jeu. Paris : Éditions de Minuit.

PICARD, M. (2002). La Tentation. Essai sur l'art comme jeu. Nîmes : J. Chambon.

RANCIÈRE, J. (2003). Le Destin des images. Paris : La Fabrique.

WIRTHNER, M. \& GARCIA-DEBANC, C. (2010). « Les savoirs des enseignants de français ». Repères.

Recherches en didactique du français langue maternelle 42, p. 5-23. En ligne : http://

journals.openedition.org/reperes/243.

\section{RÉSUMÉS}

Dans cet article, nous cherchons à documenter les représentations des professeurs stagiaires de français à travers leurs choix méthodologiques concernant la lecture de l'image en lien avec le texte littéraire ainsi que le type de difficultés qu'ils rencontrent.

En convoquant les cadres théoriques de la sémiotique post-structurale, nous nous sommes interrogées sur ce que signifie « lire » une image quand les outils préconisés par les programmes de français depuis 1985 relèvent généralement des pratiques littéraires. Quelle didactisation de la relation entre arts visuels et littérature ces programmes mettent-ils alors en œuvre ? Et quelle 
place accordent-ils à l'expression de la sensibilité et de la subjectivité du spectateur ? Une étude exploratoire (corpus de questionnaires et de séances de lecture de l'image entre 2018 et 2020 auprès de professeurs stagiaires de lettres) relativise les difficultés énoncées par les enseignants. Elle interroge également le sens des activités mises en place et leur exploitation littéraire dans le cadre de la séquence de français.

In this article we seek to document the representations of trainee French teachers through their methodological choices concerning the reading of the image in connection with the literary text as well as the type of difficulties they encounter.

By summoning the theoretical frameworks of post-structural semiotics, we wonder what it means to "read" an image when the tools advocated by French programs since 1985 generally relate to literary practices. What didactization of the relationship between visual arts and literature do these programs implement? And what place do they give to the expression of the viewer's sensitivity and subjectivity? An exploratory study (corpus of questionnaires and image reading sessions between 2018 and 2020 with trainee humanities teachers) puts the difficulties mentioned by teachers into perspective. It also questions the meaning of the activities implemented and their literary exploitation within the framework of the French sequence.

INDEX

Mots-clés : image, art, littérature, méthode d'analyse, émotion

Keywords : image, art, literature, analysis method, emotion

\section{AUTEUR}

\section{BÉNÉDICTE DUVIN-PARMENTIER}

Université Fédérale Toulouse Midi-Pyrénées, PLH, F-31000 Toulouse, France 